\title{
Virtual pyramid wavefront sensor for phase unwrapping
}

\author{
Vyas Akondi ${ }^{1,}$, Brian Vohnsen ${ }^{2}$, And Susana Marcos ${ }^{1}$ \\ ${ }^{1}$ Visual Optics and Biophotonics Laboratory, Instituto de Óptica "Daza de Valdés," Consejo Superior de Investigaciones Científicas, C/Serrano 121, 28006 \\ Madrid, Spain \\ ${ }^{2}$ Advanced Optical Imaging Group, School of Physics, University College Dublin, Belfield, Dublin 4, Ireland \\ ${ }^{*}$ Corresponding author: vyas.akondi@io.cfmac.csic.es \\ Compiled September 15, 2017
}

\begin{abstract}
Noise affects wavefront reconstruction from wrapped phase data. A novel method of phase unwrapping is proposed with the help of a virtual pyramid wavefront sensor. The method was tested on noisy wrapped phase images obtained experimentally with a digital phase-shifting point diffraction interferometer. The virtuality of the pyramid wavefront sensor allows easy tuning of pyramid apex angle and modulation amplitude. It is shown that an optimal modulation amplitude obtained by monitoring the Strehl ratio helps in achieving better accuracy. Through simulation studies and iterative estimation, it is shown that the virtual pyramid wavefront sensor is robust to random noise. (๑) 2017 Optical Society of America
\end{abstract}

OCIS codes: (010.1080) Active or adaptive optics; (010.7350) Wave-front sensing; (120.5050) Phase measurement; (280.4788) Optical sensing and sensors; (080.1010) Aberrations (global); (110.4280) Noise in imaging systems; (120.3180) Interferometry.

http://dx.doi.org/10.1364/ao.XX.XXXXXX

\section{INTRODUCTION}

Phase unwrapping is an important step in several optical interferometry, adaptive optics and imaging applications [1-3]. The effects of noise become crucial when the number of pixels per interference fringe is not sufficiently large and the signal to noise ratio is low. In such situations, conventional phase unwrapping methods are not consistent [4]. Most phase unwrapping methods are application dependent and demand an optimization of multiple parameters. The sources of noise are inherent diffraction, light source fluctuations, aberrations due to the sample and the optical system. Noise can be overcome by smartly avoiding noisy pixels in a branch cut phase unwrapping algorithm [1], although this fails if the noise is high [5-11]. Filtering is often used to reduce noise and such an operation allows smoothening near the phase jumps and elimination of potential useful information, which could adversely affect phase unwrapping [12].

Recently, a virtual Hartmann-Shack (HS) method was proposed for phase unwrapping [4]. The wrapped phase was assumed to be incident on an array of simulated microlenses. The locations of the simulated HS focal spots were estimated using the intensity weighted centroiding algorithm [13] and the unwrapped phase was recovered from the calculated local wavefront slopes. It was shown that the accuracy of phase unwrapping primarily depends on sampling of the wrapped phase and diffraction-limited wavefront sensing can be achieved using an iterative estimation procedure in the presence of noise. However, the performance of the virtual HS is limited by localized errors due to centroiding arising from random noise and has a cumulative effect on the unwrapped phase. In addition, the wrapped phase is sub-divided into a finite number of subapertures and the slope sampling is limited by the resolution of the phase map. Ambiguities due to jumps in phase close to the borders of the subapertures can cause further inaccuracies. Aforementioned shortcomings place a lower limit on the signalto-noise ratio (SNR) for which diffraction-limited performance may be achieved [14]. In this letter, a novel method of phase unwrapping is proposed based on the principles of a pyramid wavefront sensor (PWS) [15] that significantly lowers this limit. The PWS performs better averaging of random noise with a larger pupil sampling and better resolution. Here, the wrapped phase is assumed to be located in the back focal plane of a lens, which is situated at a focal distance away from a simulated pyramidal prism with four facets. Since the PWS is not physically present, this method of phase unwrapping is called the virtual PWS. Modulation plays an important role in controlling the dynamic range and sensitivity of the PWS [16] and its role in phase unwrapping is studied. The method is tested on noisy interferograms recorded experimentally with a CCD camera in a digital phase-shifting (PS) point diffraction interferometer (PDI) $[4,17]$ by using a spatial light modulator to avoid mechanically moving components. The light used was a $632.8 \mathrm{~nm}$ He-Ne laser. Aberrations are introduced with the help of a MEMS deformable mirror (Boston Micromachines Corporation ${ }^{\mathrm{TM}}$ ) in closed-loop with a commercial HS wavefront sensor. 


\section{METHOD}

Let us imagine that the wrapped phase, $\phi^{w}(x, y)$ (experimentally obtained in a PS-PDI when aberrations were induced with a deformable mirror) defined in the interval $[-\pi \pi]$ within a circular pupil, $P(x, y)$ be located at the back focal plane of a lens with a focal length of $1 \mathrm{~m}$ (physically not present) that focusses light onto a pyramidal prism (physically not present) with its phase, $T(X, Y)[16,18]$ as illustrated in Fig. 1. This pyramidal phase would divide the incident light into four distinct pupils and using a fast Fourier transform (FFT) method [19], the pupil plane intensity, $I_{p y r}$ can be evaluated as shown below:

$$
I_{p y r}(x, y)=\left|\operatorname{FFT}\left(\operatorname{FFT}\left(P(x, y) \cdot e^{i \phi^{w}(x, y)}\right) \cdot T(x, y)\right)\right|^{2}
$$

A few examples of the sources of aberrations used when recording the wrapped interferogram are imperfections in the optical elements, a patient's eye [20], a specimen under an optical microscope [21] and atmospheric turbulence in a telescope [22]. From a linear combination of the intensities in the four pupils of the virtual PWS $\left(I_{j}, 1 \leq j \leq 4\right)$ in $I_{p y r}(x, y)$, the local ' $x$ ' and ' $y$ ' wavefront slopes, $S_{x}(x, y)$ and $S_{y}(x, y)$ can be evaluated [16]. The unwrapped phase, $\phi(x, y)$ is reconstructed from the estimated slope values using the slope geometry of Southwell [13, 14, 23]. The Zernike polynomials are used to decompose the reconstructed wavefront $\phi(x, y)$ using a least square fitting technique to eliminate the high spatial frequency components and artifacts arising from noise.

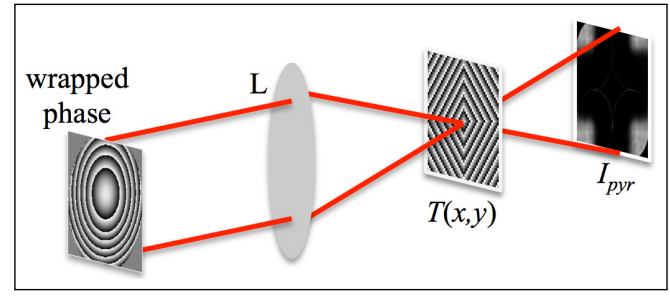

Fig. 1. Illustration of the proposed virtual pyramid wavefront sensor. Here, the lens ' $L$ ' and the pyramidal phase $T(x, y)$ are physically not present. The wrapped phase is obtained in a digital PS-PDI [17] and $I_{p y r}$ is evaluated using Eq. 1.

To compare and test the capability of the proposed method in the presence of noise, simulations are performed. The PS PDI interferograms $\left(\mathcal{I}_{1}, \mathcal{I}_{2}\right.$ and $\mathcal{I}_{3}$ corresponding to phase shifts of $0, \pi / 2$ and $\pi$ respectively) and hence the wrapped phase are calculated as follows:

$$
\phi^{w}(x, y)=\tan ^{-1}\left[\frac{2 \mathcal{I}_{2}(x, y)-\mathcal{I}_{3}(x, y)-\mathcal{I}_{1}(x, y)}{\mathcal{I}_{3}(x, y)-\mathcal{I}_{1}(x, y)}\right] .
$$

Here, the interferograms $\left(\mathcal{I}_{1-3}\right)$ in a digital PS PDI are obtained as described earlier [4,17].

Root-mean-square (RMS) of the residual wavefront is used to evaluate the quality of reconstruction [4]. Alternately, the Strehl ratio of the residual wavefront is evaluated by assuming that the pupil plane has aberrations equal to the difference of the induced aberration and the reconstructed wavefront. If the estimated wavefront is different from the induced wavefront, the Strehl ratio would be lower than unity.

\section{RESULTS}

Figure 2(a) shows the wavefronts - defocus, astigmatism, coma and secondary astigmatism - generated in closed-loop using the

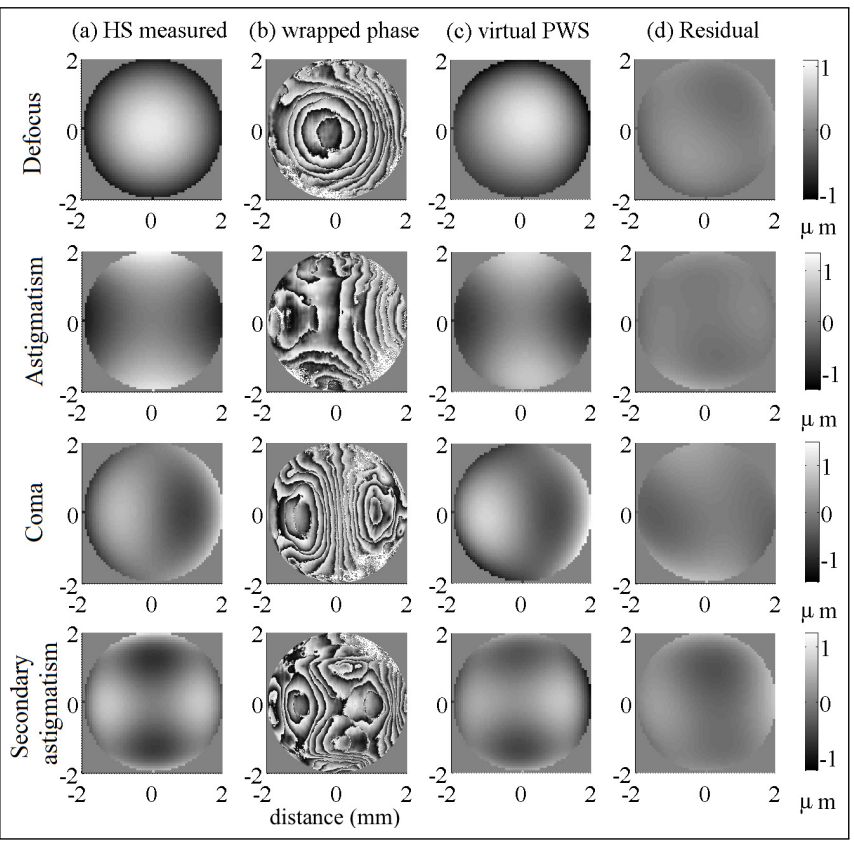

Fig. 2. Experiments: (a) wavefronts measured with a commercial HS wavefront sensor; (b) wrapped phase (radians: $-\pi$ to $\pi$ ) obtained in a PS PDI; (c) wavefront reconstructed with the virtual PWS and $(\mathrm{d})$ residual wavefront error.

deformable mirror and the HS wavefront sensor. The wrapped phase images are calculated using Eq. (2) from the interferograms recorded by a digital PS PDI and are shown in Fig. 2(b). From the wrapped phase, the intensity at the pupil plane, $I_{p y r}$ of a pyramid wavefront sensor is evaluated using Eq. (1) and 25 measurements in a circular modulation such that the tip of the pyramid oscillates in a circular path around the focal point of the focussed beam. The radius of the circular path is the modulation amplitude. Then, the local wavefront slopes, $S_{x}$ and $S_{y}$ are estimated. Finally, the wavefronts are reconstructed using the singular value decomposition technique [13]. The reconstructed wavefronts are decomposed using the first four orders of Zernike polynomials and are shown in Fig. 2(c). It may be noted that the wavefronts reconstructed with the virtual PWS match well with the induced aberrations as validated by the low residual wavefront error shown in Fig. 2(d), which is calculated by subtracting the decomposed wavefronts in Fig. 2(c) from the induced aberrations in Fig. 2(a). Here, a modulation amplitude that maximizes the estimated Strehl ratio for each aberration is used. This optimal modulation depends on the nature of the aberrations, their magnitude and SNR. Although $1529 \times 1529$ ' $x$ ' and ' $y$ ' local slope values were calculated from the wrapped phase, for reconstruction of the wavefront, the slope matrices were resized to $31 \times 31$ by using bilinear interpolation. This sampling was chosen because a higher sampling needs more computations and increasing the slope sampling beyond $31 \times$ 31 did not increase the Strehl ratio any further. On average, the Strehl ratio and RMS were found to be 0.28 and $0.14 \mu \mathrm{m}$ respectively for the residuals in Fig. 2(d), indicating a big leap in performance over virtual HS [4] that resulted in a mean Strehl ratio and mean RMS error of 0.11 and $0.20 \mu \mathrm{m}$ for the same wrapped phase images.

The estimated Strehl ratio and RMS error as a function of modulation amplitude are shown in Fig. 3. Simulations were 


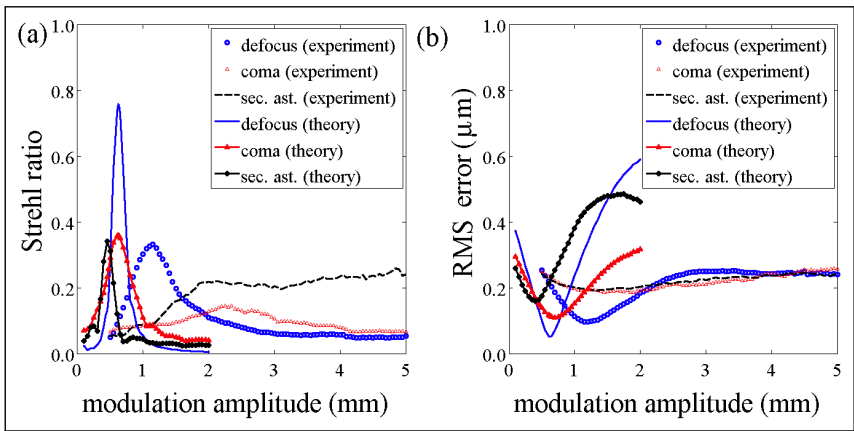

Fig. 3. Effect of modulation amplitude on wavefront sensing accuracy in the experiments and theoretical predictions at SNR $=\infty$ in terms of (a) Strehl ratio and (b) RMS error.

performed at $\mathrm{SNR}=\infty$ and clearly, the accuracy is higher under noise-free conditions. In addition, the experimental curves are broader than the theoretical counterparts. The differences arise due to inaccuracies in the generation of the aberrations, misalignment errors in the digital PS PDI and the presence of noise in the interferograms. The tilt due to PS (as seen in Fig. 2(b)) prevents convergence with increasing modulation as predicted earlier [16].

Figure 4 shows the results of simulations using the virtual PWS. Here, the wavefronts measured by the HS wavefront sensor were used as the starting point in the simulations (see Fig. 4(a)) and no effects of noise were included while estimating the wrapped phase shown in Fig. 4(b) from the calculated interferograms $[4,17]$. The wavefronts reconstructed with the virtual PWS from the wrapped phase in Fig. 4(b) are shown in Fig. 4(c). The mean RMS error shown in Fig. 4(d) is $0.10 \mu \mathrm{m}$ and the best accuracy was achieved near a modulation of $0.5 \mathrm{~mm}$ (see Fig. 3).

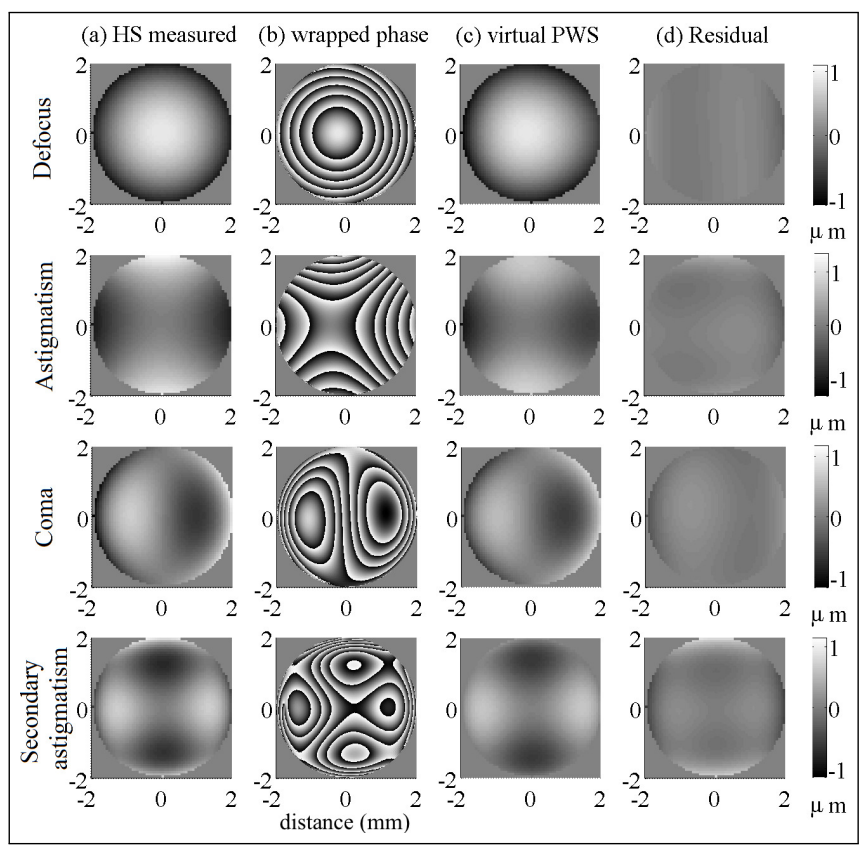

Fig. 4. Simulations: (a) wavefronts measured with a commercial HS wavefront sensor; (b) simulated wrapped phase (radians: $-\pi$ to $\pi$ ) in a PS PDI; (c) wavefront reconstructed with the virtual PWS and (d) residual wavefront error.
To study the effects of noise through simulations, independently generated white Gaussian noise was added to each of the interferograms $\left(\mathcal{I}_{1-3}\right)$ before calculating the wrapped phase (see Eq. (2)). As the SNR increases, the discrepancies in the detected slopes reduce resulting in an increase in the Strehl ratio of the residual wavefront map as illustrated by the simulation results in Fig. 5(a) while sensing the aberrations introduced by the deformable mirror (see Fig. 2(a)). With an increase in the magnitude of aberrations or with the inclusion of higherorder aberrations, the minimum SNR needed for convergence increases. This is attributed to an increase in the number of fringes per pixel. However, for a fixed modulation amplitude (optimal value predicted at $\mathrm{SNR}=\infty$ ) and following an iterative approach while estimating Strehl ratio $[4,16]$, it is possible to improve performance as illustrated in Fig. 5(b). This is done by assuming that the residual wavefront obtained in the first loop of the phase unwrapping process is the new wavefront for the second iteration. This new wavefront is used to calculate the interferograms and the wrapped phase for the second iteration and so on. This iterative procedure can be implemented in an optical system by monitoring the point spread function with a CCD camera. It can be seen in Fig. 5(b) that for the case of defocus, a decrease in the SNR from $10 \mathrm{~dB}$ to $5 \mathrm{~dB}$ requires an increase in the minimum number of iterations by three to go beyond the diffraction limit. Since most applications including visual optics

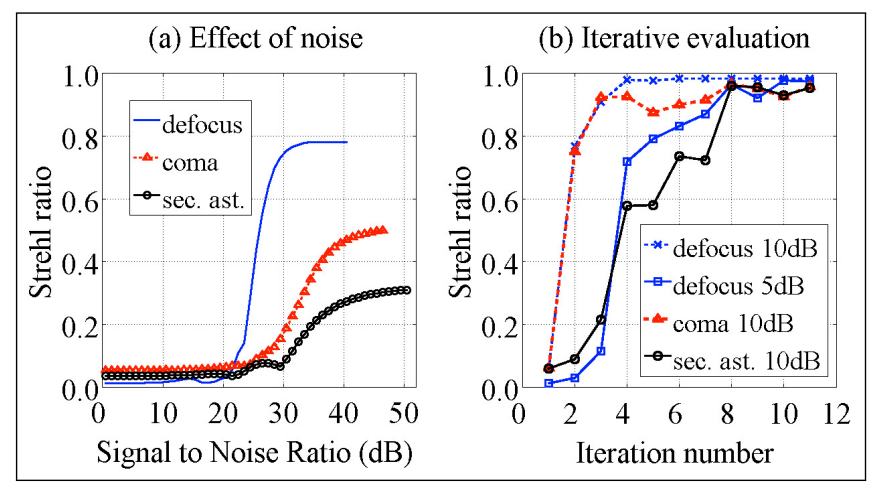

Fig. 5. Simulations: (a) Accuracy improves with increasing signal while sensing simulated aberrations (Fig. 4(a)) and results of (b) iterative evaluation. Here, random noise (single trial) is added to the calculated interferograms.

involve a combination of the low-order aberrations that play a prominent role, the proposed method was tested with randomly simulated wavefronts containing up to three orders of Zernike polynomials excluding the piston and the two tilt terms. On average, the peak-to-valley of the randomly simulated aberrations is $2.3 \mu \mathrm{m}$ and the chosen modulation amplitude is $0.5 \mathrm{~mm}$ (sensitivity improves with smaller modulation and the lower limit is given by the number of iterations for convergence). The modulation amplitude is progressively reduced with increasing iteration number to avoid over-estimation. The performance of the virtual PWS is compared with virtual HS [4] and Fourier [24] phase unwrapping methods in the presence of noise. In the case of the virtual HS, reconstruction was performed with $20 \times 20$ subapertures and $32 \times 32$ pixels were used for centroiding the virtual HS focal spots. An identical slope sampling of $20 \times 20$ was used in the case of the virtual PWS for comparison. This sampling was chosen to compare against the optimal sampling in a virtual HS when sensing aberrations with a digital PS PDI 
[4]. At SNR $=10 \mathrm{~dB}$, the Fourier phase unwrapping algorithm fails [4] and the virtual PWS is evidently superior and saturates at a higher Strehl ratio value in comparison with the virtual HS for cases of $10 \mathrm{~dB}$ and $5 \mathrm{~dB}$ SNR (Fig. 6), illustrating the potential of the virtual PWS at very low SNR. The error bars for each iteration shown in Fig. 6 indicate the standard deviation of the Strehl ratio values of ten independently generated random wavefronts. Likewise, in the case of high-order aberrations and their random linear combinations, the virtual PWS consistently converged to higher Strehl ratios in the presence of random noise.

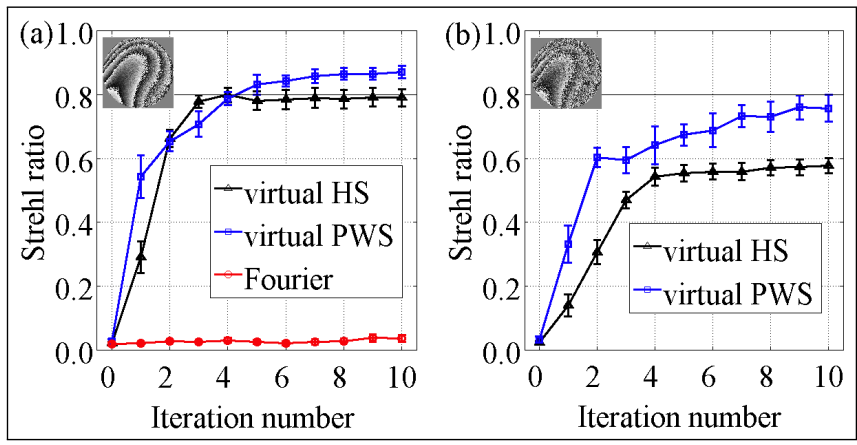

Fig. 6. Comparison of phase unwrapping methods at (a) $10 \mathrm{~dB}$ and (b) $5 \mathrm{~dB}$ while sensing ten randomly simulated wavefronts. Corresponding sample wrapped phase maps are shown within the plots.

The method was also tested on a randomly simulated atmospheric turbulence phase screen for a $1 \mathrm{~m}$ class telescope on a 125 x 125 grid [16] as shown in Fig. 7(a). Its high spatial frequency components result in a challenging wrapped phase shown in Fig. 7(b). The reconstructed wavefront (Fig. 7(c)) obtained by applying the virtual PWS resulted in a residual (Fig. 7(d)) with a Strehl ratio of 0.88 and RMS error of $0.05 \mu \mathrm{m}$ after four iterations when $61 \times 61$ slope sampling is used. Here, Zernike decomposition is not applied to avoid eliminating vital high-frequency information.

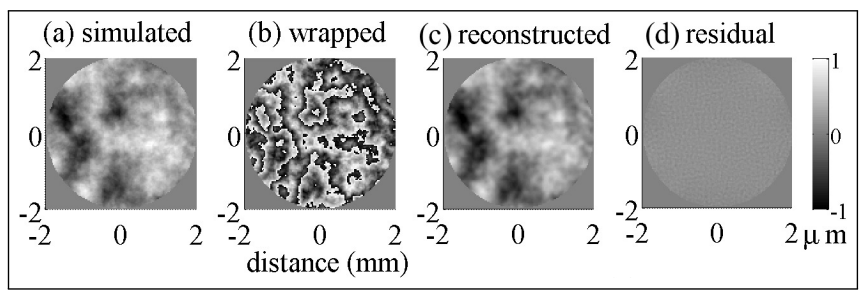

Fig. 7. (a) Simulated turbulence phase screen (b) Wrapped phase corresponding to (a) in the interval $[-\pi \pi]$; (c) reconstructed with the virtual PWS (d) residual wavefront error.

\section{DISCUSSION}

Focal spot centroiding influences the reconstruction accuracy of HS wavefront sensors [25]. The centroiding errors arising from noise in the wrapped images lead to inaccuracies in the virtual HS method [4] and the estimated Strehl ratio drops with increasing noise [14]. Furthermore, the total number of subapertures needs to be increased to compensate for a decrease in SNR and in order to retain the same number of pixels per subaperture (and not compromise centroiding accuracy), the effective pupil diameter is a larger matrix. This makes the virtual HS method slow and computationally challenging for low SNR. The virtual PWS is relatively robust and the 4 pupil intensity images enable a direct evaluation of global wavefront slopes and does not need local wavefront slope estimation that may involve additional errors as is the case with the virtual HS. The size of the pupils can be adjusted to the number of slope measurements needed for phase unwrapping. And, by controlling the apex angle of the pyramidal prism, the pupils can be placed right next to one another in a $2 \times 2$ grid. For instance, to compute $31 \times 31$ slope values, an intensity matrix, $I_{p y r}$ with $62 \times 62$ pixels is sufficient to estimate the wavefronts. In comparison, a virtual HS uses $\sim 20 \times 20$ pixels per subaperture and to obtain $31 \times 31$ slope values, it requires to compute an intensity matrix of dimension $620 \times 620$ pixels, ten times larger than the matrix needed in a virtual PWS. In the absence of noise, the virtual HS and virtual PWS gave similar results with minor differences in the residual wavefronts.

Modulation amplitude has an important role in controlling the dynamic range and sensitivity of a pyramid wavefront sensor [26]. It was shown earlier that the optimal modulation amplitude increases in the presence of noise [27]. The same can be noted in Fig. 3. In addition, a decrease in the magnitude of the aberrations leads to a decrease in the optimal modulation amplitude [16] and hence the modulation amplitude is premeditatedly reduced with increasing iterations as the residual decreases. In practical situations, optimal modulation can be obtained by minimizing the difference between the wrapped phase image and the rewrapped image obtained from the reconstructed wavefront. The accuracy can be improved further by increasing the number of facets in a pyramid wavefront sensor [27]. In addition, increasing the number of phase-shifts in a PS PDI, the measurement noise can be reduced. The conclusions derived with Strehl ratio as an evaluation metric in Figs. 5 and 6 were not affected when RMS error is used.

The virtuality of the PWS that is proposed here is unaffected by the practical limitations of a physical PWS including the need for moving parts to achieve modulation, the precise alignment of the tip of the pyramidal prism at the focus of a lens and manufacturing defects, all of which can adversely affect recovery of the aberrations. There exist non-moving solutions for closedloop operation [28]. However, they need several iterations to achieve diffraction-limited point spread function [16].

In conclusion, a novel method of phase unwrapping called the virtual PWS has been proposed. Although the virtual PWS is tested on wrapped phase images in a PS PDI, the efficacy of the method is not limited to this application. It can be easily adapted to any complex phase unwrapping application including digital holographic microscopy, sample motion-detection in spectral domain optical coherence tomography and other medical imaging modalities such as magnetic resonance elastography.

Funding. The research leading to these results has received funding from Consejería de Educación, Juventud y Deporte of Comunidad de Madrid and the People Programme (Marie Curie Actions) of the European Union's Seventh Framework Programme (FP7/2007-2013) under REA grant agreement $\mathrm{n}^{\circ} 291820$ to VA; Science Foundation Ireland (grants: 07/SK/B1239a and 08/IN.1/B2053); University College Dublin (seed funding: SF665) to BV; ERC Grant Agreement [ERC-2011-AdG 294099] and Spanish Government Grant FIS2014-56643-R to SM. 


\section{REFERENCES}

1. R. M. Goldstein, H. A. Zebker and C. L. Werner, "Satellite radar interferometry: Two-dimensional phase unwrapping," Radio Sci. 23(4), 713-720 (1988).

2. D. L. Fried, "Adaptive optics wave function reconstruction and phase unwrapping when branch points are present," Opt. Commun. 15, 43-72 (2001).

3. B. J. Vakoc, S. H. Yun, J. F. de Boer, G. J. Tearney and B. E. Bouma, "Phase-resolved optical frequency domain imaging," Opt. Express 13(14), 5483-5493 (2005).

4. V. Akondi, C. Falldorf, S. Marcos and B. Vohnsen, "Phase unwrapping with a virtual Hartmann-Shack wavefront sensor," Opt. express 23(20), 25425-25439 (2015).

5. M. A. Navarro, J. C. Estrada, M. Servin, J. A. Quiroga and Javier Vargas, "Fast two-dimensional simultaneous phase unwrapping and low-pass filtering," Opt. Express 20(3), 2556-2561 (2012).

6. S. Heshmat, S. Tomioka and S. Nishiyama, "Performance evaluation of phase unwrapping algorithms for noisy phase measurements," Int. J. Optomechatronics, 8(4), 260-274 (2014).

7. M. A. Herráez, D. R. Burton, M. J. Lalor, and D. B. Clegg, "Robust, simple, and fast algorithm for phase unwrapping," Appl. Opt. 35(29), 5847-5852 (1996).

8. J. M. Huntley, "Noise-immune phase unwrapping algorithm," Appl. Opt. 28(16), 3268-3270 (1989).

9. J. M. Huntley and H. Saldner, "Temporal phase unwrapping algorithm for automated interferogram analysis," Appl. Opt. 32(17), 3047-3052 (1993).

10. J. A. Quiroga and E. Bernabeu, "Phase-unwrapping algorithm for noisy phase-map processing," Appl. Opt. 33(29), 6725-6731 (1994)

11. R. Cusack, J. M. Huntley, and H. T. Goldrein, "Improved noise-immune phase-unwrapping algorithm," Appl. Opt. 34(5), 781-789 (1995).

12. J-S. Lee, K. P. Papathanassiou, T. L. Ainsworth, M. R. Grunes, and A. Reigber, "A new technique for noise filtering of SAR interferometric phase images," IEEE Trans. Geosci. Remote Sens. 36(5), 1456-1465 (1998).

13. R. M. Basavaraju, V. Akondi, S. J. Weddell and R. P. Budihal, "Myopic aberrations: Simulation based comparison of curvature and Hartmann Shack wavefront sensors," Opt. Commun. 312, 23-30 (2014).

14. V. Akondi and B. Vohnsen, "Myopic aberrations: impact of centroiding noise in Hartmann Shack wavefront sensing," Ophthalmic Physiol. Opt. 33(4), 434-443 (2013).

15. R. Ragazzoni, "Pupil plane wavefront sensing with an oscillating prism," J. Mod. Opt. 43(2), 289-293 (1996).

16. V. Akondi, S. Castillo and B. Vohnsen, "Digital pyramid wavefront sensor with tunable modulation," Opt. Express 21(15), 18261-18272 (2013).

17. V. Akondi, A. R. Jewel and B. Vohnsen, "Digital phase-shifting point diffraction interferometer," Opt. Letts. 39(6), 1641-1644 (2014).

18. V. Korkiakoski, C. Vèrinaud, M. Le Louarn, and R. Conan, "Comparison between a model-based and a conventional pyramid sensor reconstructor," Appl. Opt. 46, 6176-6184 (2007).

19. C. Vèrinaud, "On the nature of the measurements provided by a pyramid wave-front sensor," Optics Communications 233, 27-38 (2004).

20. V. Akondi, A. R. Jewel, and B. Vohnsen, "Closed-loop adaptive optics using a spatial light modulator for sensing and compensating of optical aberrations in ophthalmic applications," J. Biomed. Opt. 19(9), 096014 (2014).

21. T. J. Gould, D. Burke, J. Bewersdorf, and M. J. Booth, "Adaptive optics enables 3D STED microscopy in aberrating specimens," Opt. Express 20(19), 20998-21009 (2012).

22. B. L. Ellerbroek, "First-order performance evaluation of adaptive-optics systems for atmospheric-turbulence compensation in extended-fieldof-view astronomical telescopes," J. Opt. Soc. Am. A 11(2), 783-805 (1994).

23. W. H. Southwell, "Wave-front estimation from wave-front slope measurements," J. Opt. Soc. Am. 70(8), 998-1006 (1980).

24. M. D. Pritt and J. S. Shipman, "Least-squares two-dimensional phase unwrapping using FFT's," IEEE Trans. Geosci. Remote Sens 32(3), 706708 (1994).
25. V. Akondi, R. M. Basavaraju and R. P. Budihal, "Centroid detection by Gaussian pattern matching in adaptive optics," Int. J. Comput. Appl. 26(1), 30-35 (2010).

26. A. Burvall, E. Daly, S. Chamot, and C. Dainty, "Linearity of the pyramid wavefront sensor," Opt. Express 14(25), 11925-11934 (2006).

27. V. Akondi, S. Castillo and B. Vohnsen, "Multi-faceted digital pyramid wavefront sensor," Opt. Commun. 323, 77-86 (2014).

28. R. Ragazzoni, E. Diolaiti, and E. Vernet, "A pyramid wavefront sensor with no dynamic modulation," Opt. Commun. 208, 51-60 (2002). 